International Journal of Power Electronics and Drive System (IJPEDS)

Vol. 10, No. 4, December 2019, pp. 1906 1913

ISSN: 2088-8694, DOI: 10.11591/ijpeds.v10.i4.pp1906-1913

\title{
Wireless power transfer through metal using inductive link
}

\author{
Tuan Anh Vu' ${ }^{1}$, Chi Van Pham², Anh-Vu Pham ${ }^{3}$, Christopher S. Gardner \\ ${ }^{1,2,3}$ Department of Electrical and Computer Engineering, University of California, Davis, United States \\ ${ }^{4}$ Lawrence Livermore National Laboratory, Livermore, United States \\ ${ }^{1}$ VNU University of Engineering and Technology, Vietnam
}

\section{Article Info}

\section{Article history:}

Received March 30, 2019

Revised April 20, 2019

Accepted May 30, 2019

\section{Keywords:}

Wireless power transfer

ABSTRACT

This paper presents a highly efficient power transfer system based on a co-design of a class-E power amplifier (PA) and a pair of inductively coupled Helical coils for through-metal-wall power transfer. Power is transferred wirelessly through a 3.1-mm thick aluminum barrier without any physical penetration and contact. Measurement results show that the class-E PA achieves a peak power gain of $25.2 \mathrm{~dB}$ and a maximum collector efficiency of $57.3 \%$, all at $200 \mathrm{~Hz}$. The proposed system obtains a maximum power transfer efficiency of $9 \%$ and it can deliver $5 \mathrm{~W}$ power to the receiver side through the aluminum barrier.
\end{abstract}

Inductive coupling

Through metal

Class-E

Power amplifier

Helical coil

\section{Corresponding Author:}

Tuan Anh Vu,
Copyright 2019 Insitute of Advanced Engineeering and Science. 口CC.png

VNU University of Engineering and Technology,

144 Xuan Thuy Rd., Cau Giay Dist., Hanoi, Vietnam.

Tel: +84-4-3754-9338

Email: tanhvu@vnu.edu.vn

\section{INTRODUCTION}

Design and optimization of wireless power transfer (WPT) systems have been well studied over the last decades to charge cell phones, electric vehicles, and power up sensors over a short distance. In recent years, there has been a great demand for wirelessly powered sensors to support structural health monitoring (SHM) for industrial applications containing metal walls and enclosures (e.g., naval vessel, aircraft, vehicle armor, metal pile and chemical vat). Traditionally, metal wall penetrations are used to feed through wires. However, drilling holes for feeding wires reduces the integrity of the structure and also causes practical issues such as the probability of leakage of toxic chemicals, loss of pressure or vacuum. The SHM systems typically require embedded sensors for data acquisition, wireless communication, and energy harvesting. These sensors need to be powered and controlled wirelessly through a metal wall without physical penetration through mechanical structures.

Several solutions have been proposed to fulfill through-metal-wall power transfer [1]. These solutions can be categorized into two main methods. The first method exploits ultrasound waves including piezoelectric transducer (PZT) [2,3] and electromagnetic acoustic transducer (EMAT) based approach [4]. Ultrasonic waves can propagate easily through a variety of metallic materials. The PZT-based system is by far the most widely used for through-metal-wall power transfer due to the high power transfer efficiency and the possibility of miniaturizing the design. The main limitation of the PZT-based system is that it requires direct coupling of piezoelectric transducers with metal wall to provide a good acoustic transmission path. In addition, the performance of the PZT-based system is highly reliant on the quality of the coupling. Poor coupling will introduce severe impedance mismatch over the acoustic-electric channel and cause the power transfer efficiency to decrease rapidly. EMAT is a non-contact approach but the power transfer efficiency is too low, which makes 
it unsuitable for highly efficient power transfer through metal wall. The second method is based on electromagnetic coupling involving inductive coupling [4,5], capacitive coupling [6,7] and magnetic resonance coupling [8]. The biggest advantage of electromagnetic coupling method is that there is no need for the transmitter and receiver to contact directly with the metal wall. The inductive power transfer (IPT) is probably the most popular approach based on electromagnetic coupling principles. The inductive coupling approach is suitable for applications with low conductivity, low permeability materials such as aluminum or stainless steel walls. It works by magnetic field coupling using a pair of inductive coils. As the skin depth of the metallic material increases rapidly with the increase of frequency, IPT systems operate at super low frequencies to minimize the influences of the Faraday shielding effects.

In this paper, a fully functional IPT system based on a co-design of a class-E PA and a pair of inductive Helical stacked coils for through-metal-wall power transfer will be presented. Measurement results show that the system obtains a peak power transfer efficiency of $9 \%$ and it is able to deliver $5 \mathrm{~W}$ power to the receiver side through the $3.1-\mathrm{mm}$ thick aluminum barrier.

\section{SYSTEM OVERVIEW}

The concept of IPT is similar to the principle of transformers in which an alternative magnetic field in primary coil induces a load voltage on secondary coil when these two coils are tightly coupled [9]. The metallic medium restricts the power transfer due to losses causing by the shielding effect or skin effect. As a result, a specific frequency range is used to overcome the skin-depth limitation of metal thicknesses such as the metal tank layer using $30-300 \mathrm{~Hz}$ range and underground pipe using $50 \mathrm{~Hz}$. For a $3.1 \mathrm{~mm}$ thick aluminum $(\sigma=38 \mathrm{MS} / \mathrm{m})$, its skin depth is $2.62 \mathrm{~mm}$ and $0.34 \mathrm{~mm}$ at $1 \mathrm{kHz}$ and $60 \mathrm{kHz}$, respectively. Therefore, the super low frequency, $f_{0}=200 \mathrm{~Hz}$, has been used as the operating frequency for the proposed system. As shown in Figure 1, the IPT system consists of a class-E PA, a pair of inductive coils, a seriesparallel LC resonant circuit and a rectifier. The power link includes two inductive coils placed on both sides of a aluminum barrier. The transmitting (Tx) and receiving ( $\mathrm{Rx}$ ) coils are represented by the inductors $L_{1}$ and $L_{2}$ while their parasitic series resistances are $R_{1}$ and $R_{2}$, respectively. The system delivers AC power to the $\mathrm{Rx}$ coil, which needs to be terminated with an optimal load, $R_{\mathrm{L}}$, to achieve the maximum power transfer. $L_{2}$ and $C_{1}$ form a series-parallel resonant circuit. When the parallel resonator is used as a receiver, the voltage drop loss in the following rectifier is small because of the high load voltage. The AC voltage is converted into the DC voltage by a full-bridge rectifier GBPC $3502 \mathrm{~W}$ from ON Semiconductor. A smoothing capacitor, $C_{2}$, is used in conjunction with the rectifier. It smooths out the fluctuation in the rectified signal to make a steady DC voltage for sensing and communication operation on the receiver side.

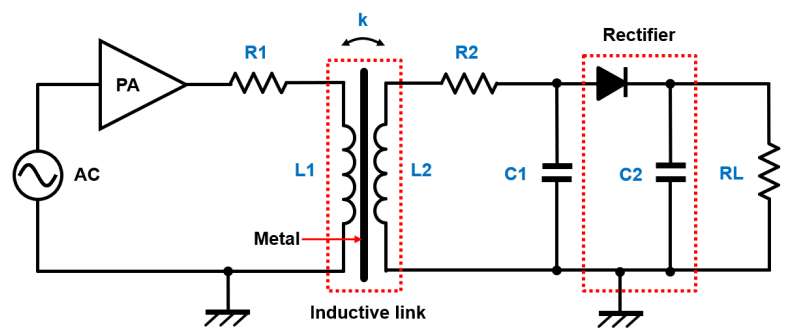

Figure 1. The proposed through-metal-wall power transfer system.

\subsection{Helical stacked coil}

The inductive link uses a pair of Helical stacked coils as shown in Figure 2. By co-axially aligning a pair of coils, the wireless IPT channel is formed without any physical penetration through the metal barrier [10]. The Helical circular geometry results in a more uniform magnetic field distribution that significantly improves the efficiency of energy transfer compared to that of the conventional Solenoid counterpart. The Helical stacked coils are constructed using American Wire Gauge (AWG) 17 magnet wire. Both coils use ferrites to increase their magnetic fields. Table 1 gives parameters of the two coils. The Tx and Rx coil inductances are $20.1 \mathrm{mH}$ and $29.6 \mathrm{mH}$, respectively. Their quality factors are 8.15 and 10.22 at the operating frequency of $200 \mathrm{~Hz}$. The coil inductances without using ferrite are $3.62 \mathrm{mH}$ and $3.9 \mathrm{mH}$. Ferrites have great effects in boosting up the coil inductances. Consequently, the quality factors of the Helical coils are improved significantly. 


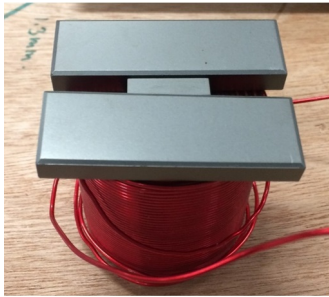

(a)

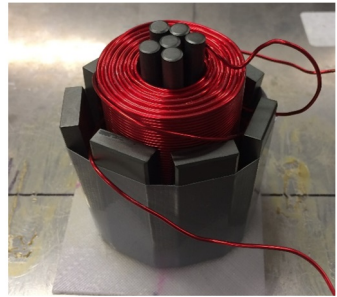

(b)

Figure 2. (a) Transmitting and (b) receiving Helical stacked coils.

Table 1. Coil design parameters

\begin{tabular}{lcc}
\hline Parameter & Tx Coil & Rx Coil \\
\hline Number of Turns & 514 & 635 \\
Inner Radius & $21 \mathrm{~mm}$ & $20 \mathrm{~mm}$ \\
Outer Radius & $49 \mathrm{~mm}$ & $47 \mathrm{~mm}$ \\
Inductance & $20.1 \mathrm{mH}$ & $29.6 \mathrm{mH}$ \\
Parasitic Resistance & $3.1 \Omega$ & $3.65 \Omega$ \\
Quality Factor & 8.15 & 10.22 \\
\hline
\end{tabular}

Power transfer efficiency (PTE) is determined by the quality factors of the inductive coils and the coupling factor $\mathrm{k}$. The product $k^{2} Q_{l} Q_{2}$ is desirable to be as high as possible. However, the shielding effect of the aluminum barrier or the metal thickness will severely degrade the coupling coefficient. Whereas, the proximity effect, or air distance from each coil to the metal surface, will reduce the quality factor of the coils. The product $k^{2} Q_{l} Q_{2}$ experiences a maximum and consequently determines the peak efficiency for an optimal operating frequency range. Therefore, design of a high-quality factor inductive coil in a small form factor is a key aspect in achieving the high efficiency power transfer. The maximum PTE of the system is calculated as follow [11]:

$$
\eta \approx \frac{1}{2+\frac{4}{k^{2} Q_{1} Q_{2}}}=\frac{1}{2+\frac{4 R_{1} R_{2}}{\omega_{0} M^{2}}}
$$

where $Q_{1}=\omega_{0} L_{1} / R_{1}$ and $Q_{2}=\omega_{0} L_{2} / R_{2}$ are the quality factors of the Tx and Rx coils, and $M$ is their mutual inductance.

Table 2. Class-E PA design parameters

\begin{tabular}{lc}
\hline Component & Value \\
\hline$C_{0}$ & $1 \mathrm{mF}$ \\
$L_{0}$ & $1.88 \mathrm{mH}$ \\
$R_{\mathrm{g} 1}$ & $1 \mathrm{k} \Omega$ \\
$R_{\mathrm{g} 2}$ & $10 \mathrm{k} \Omega$ \\
$C_{\mathrm{sh}}$ & $0.6 \mathrm{mF}$ \\
$C_{\mathrm{s}}$ & $33 \mu \mathrm{F}$ \\
\hline
\end{tabular}

\section{2. $200 \mathrm{~Hz}$ Class-E Power Amplifier}

A class-E PA is co-designed with the inductive link for power transfer. Class-E PAs achieve significantly higher efficiency than conventional class-B or - $\mathrm{C}$ counterparts. The efficiency is maximized by minimizing power dissipation, while providing a desired output power. The insulated-gate bipolar transistor (IGBT) STGW15S120DF3 from STMicroelectronics has been used as the power transistor. This IGBT family has been specifically optimized for low switching frequencies. Figure 3 shows the schematic of the $200 \mathrm{~Hz}$ class-E PA and its component values are given in Table 2. The PA design is obtained by the well-known Sokal-Raab approach $[12,13]$. In this design, the IGBT operates as an on/off switch and the load network shapes the voltage and current waveforms to prevent simultaneous high voltage and high current in the transistor. As a result, the power dissipation is minimized, especially during the switching transitions. $C_{0}$ is the coupling capacitor at the input of the PA. $R_{\mathrm{g} 1}$ and $R_{\mathrm{g} 2}$ form a voltage divider for gate biasing. The DC-feed choke, $L_{0}$ provides the 
connection to the DC power supply. The IGBT is driven with a gate voltage of $8 \mathrm{~V}$ and a supply voltage of $20 \mathrm{~V}$. The load network is designed considering effects of the inductive link. The inductances of the Helical coils become a part of the load network. The initial values for the shunt and series capacitors, $C_{\mathrm{sh}}$ and $C_{\mathrm{s}}$, are calculated by the following equations:

$$
\begin{gathered}
C_{\mathrm{sh}}=\frac{1}{34.22 f_{0} R_{\mathrm{L}}}\left(1+\frac{0.91}{Q_{\mathrm{L}}}-\frac{1.03}{Q_{\mathrm{L}}{ }^{2}}\right) \\
C_{\mathrm{s}}=\frac{1}{2 \pi f_{0} R_{\mathrm{L}}}\left(\frac{1}{Q_{\mathrm{L}}-0.1}\right)\left(1+\frac{1.01}{Q_{\mathrm{L}}-1.79}\right)
\end{gathered}
$$

where $Q_{\mathrm{L}}$ is the loaded quality factor of the load network.

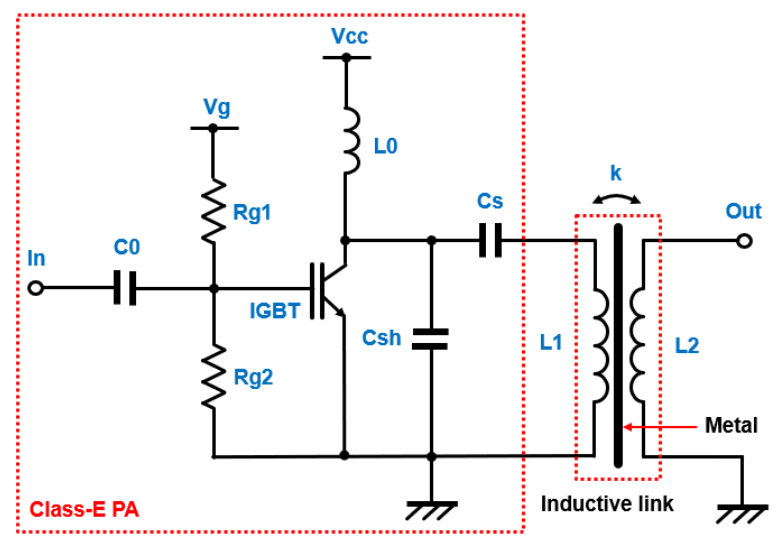

(a)

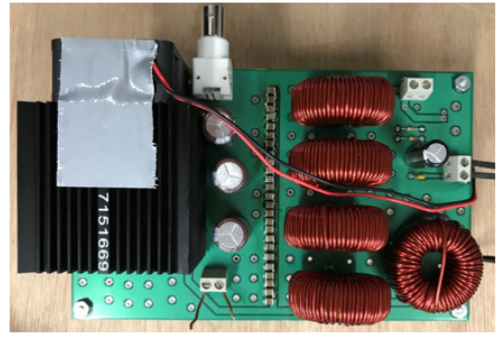

(b)

Figure 3. (a) Schematic and (b) fabricated class-E power amplifier .

\section{EXPERIMENTAL RESULTS}

A complete prototype was built to demonstrate the effectiveness of the proposed system. Figure 4 illustrates the measurement setup for power transfer through a $400 \mathrm{~mm} \times 400 \mathrm{~mm} \times 3.1 \mathrm{~mm}$ aluminum barrier. The Tx coil cannot be seen in the figure since it is covered by the aluminum barrier. Figure 5 shows the measured collector voltage and collector current waveforms in the time domain captured by an oscilloscope. The voltage and current switching transitions are time-displaced from each other.

Figure 6 shows a typical measured output signal spectrum of the class-E PA in the frequency domain captured by a vector signal analyzer. The PA exhibits a good linearity when most of the power is at the fundamental frequency. At $200 \mathrm{~Hz}$, the power of transmitted signal is $43.2 \mathrm{dBm}$. Harmonic output of the the class-E amplifier is similar to that of the class-B counterpart. The strongest harmonic is the second one. Without filtering, the second-harmonic power is $-17 \mathrm{dBc}$ ( $2 \%$ of the fundamental power) and the third-harmonic power is $-26.4 \mathrm{dBc}(0.23 \%$ of the fundamental power $)$. 


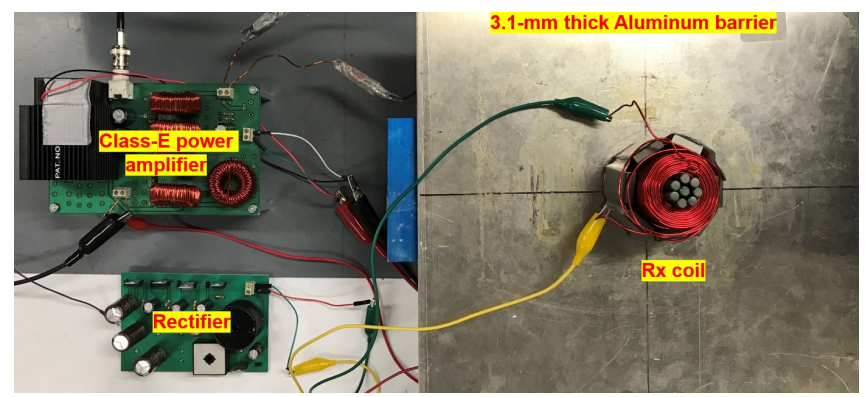

Figure 4. Measurement setup for through-metal-wall power transfer.

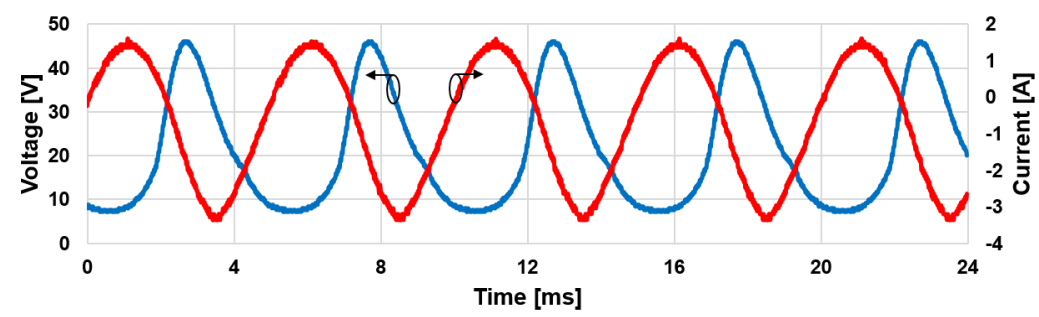

Figure 5. Measured collector voltage vs. collector current of the class-E PA.

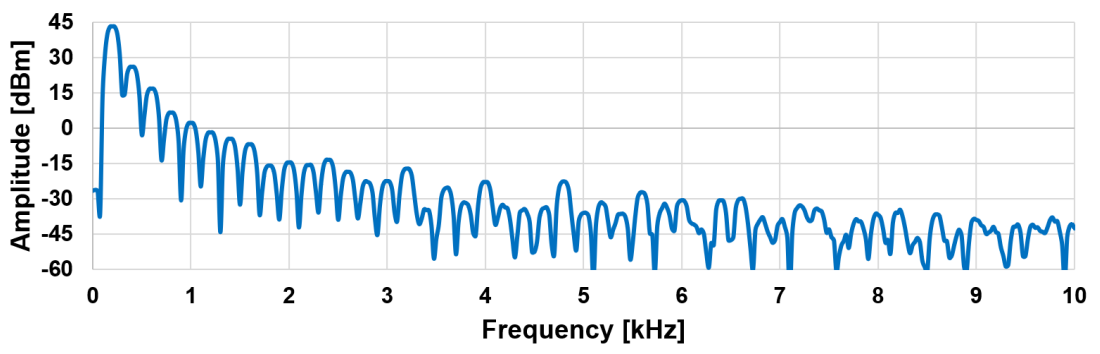

Figure 6. Measured output signal spectrum of the class-E PA.

Figure 7 shows the measured gain and output power versus the input powers of the class-E PA. It archives a peak gain of $25.2 \mathrm{~dB}$ at $21.7 \mathrm{dBm}$ input power. The output power of the PA increases linearly with the input power. At $24.2 \mathrm{dBm}$ input power, the output power is $48.5 \mathrm{dBm}$ or $70.8 \mathrm{~W}$. The measured collector efficiency as a function of the output power is shown in Figure 8. The PA obtains a peak efficiency of 57.3\% at $42.2 \mathrm{~W}$ output power. The efficiency decrease slowly with increasing output powers. At $70.8 \mathrm{~W}$ output power, the efficiency is $50 \%$. The lower value of PA efficiency can be explained by the reduced influences of ferrites at high output powers. Figure 9 shows the measured PTE and received power versus output power of the proposed IPT system. The power transfer system obtains the peak PTE of $9 \%$ and it can deliver $5 \mathrm{~W}$ power to the receiver side through the $3.1-\mathrm{mm}$ thick aluminum barrier.

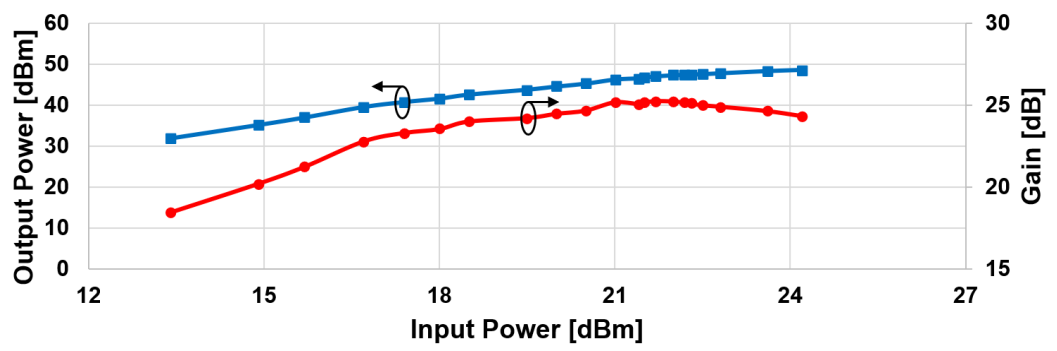

Figure 7. Measured gain and output power of the class-E PA. 


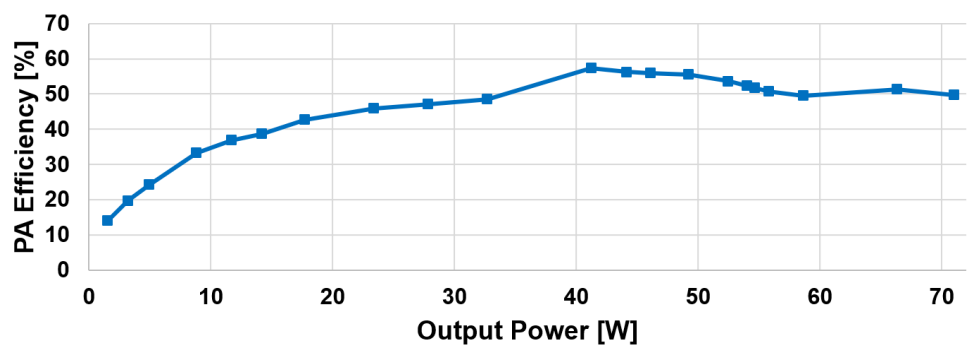

Figure 8. Measured efficiency of the class-E PA.

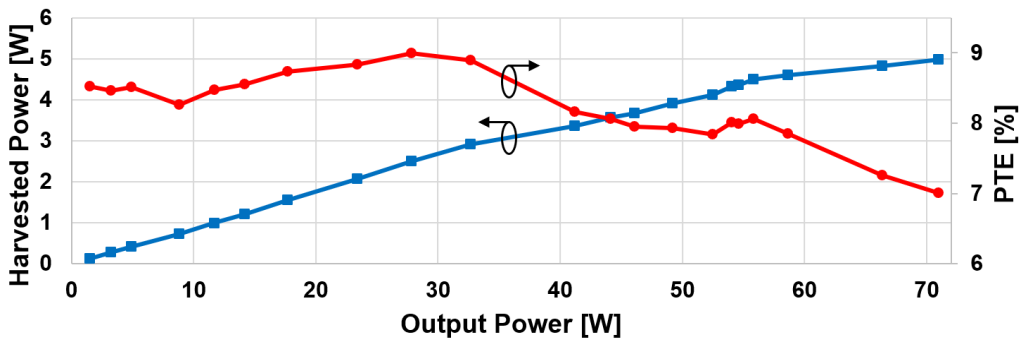

Figure 9. Measured power transfer efficiency and received power.

Table 3 summarizes the performance of the proposed IPT system and compares it to other published through-metal-wall power transfer systems based on electromagnetic coupling. Graham et al. [4] transferred power through a $20 \mathrm{~mm}$ thick, $130 \mathrm{~mm}$ diameter stainless steel disc of a relative permeability of $\mu_{\mathrm{r}}=1.1$. The results showed that an efficiency exceeding $4 \%$ can be achievable with an operating frequency of $500 \mathrm{~Hz}$. However, when the relative permeability of the metal material is increased to $\mu_{\mathrm{r}}=10$, the peak achievable efficiency is only $0.2 \%$. Zangl et al. [5] investigated the feasibility of power delivery through a tin container. The experiment results have shown that at least $30 \mu \mathrm{W}$ of power can be transferred through the 5-mm thick tin container to power the fill level measurement capacitive sensor circuit using a continuous wave carrier signal at $50 \mathrm{~Hz}$. However no information on PTE was presented in the paper. Yamakawa et al. [8] reported their work on wireless power transmission into a space enclosed by metal walls using magnetic resonance coupling. The results have shown that a $1.2 \mathrm{~W}$ of electric power can be supplied to LEDs through a stainless steel wall 5-mm thick with an efficiency of $10 \%$ over a transmission distance of $10 \mathrm{~cm}$.

Table 3. Comparison with previous published through-metal-wall power transfer systems

\begin{tabular}{lcccc}
\hline Design & {$[4]$} & {$[5]$} & {$[8]$} & This work \\
\hline Frequency & $400 \mathrm{~Hz}$ & $50 \mathrm{~Hz}$ & $50 \mathrm{~Hz}$ & $\mathbf{2 0 0} \mathbf{H z}$ \\
Coil Design & Loop Solenoid & Solenoid & Solenoid & Helical \\
System Topology & Inductive coupling & Inductive coupling & Mag. reson. coupling & Inductive coupling \\
Metal Thickness & Stainless steel-20 mm & Tin-5 mm & Stainless steel-5 mm & Aluminum-3.1 mm \\
Distance & $0 \mathrm{~cm}$ & $0 \mathrm{~cm}$ & $10 \mathrm{~cm}$ & $\mathbf{0 ~ c m}$ \\
Received Power & - & $30 \mu \mathrm{W}$ & $1.2 \mathrm{~W}$ & $\mathbf{5 ~ W}$ \\
PTE & $4 \%$ & - & $10 \%$ & $\mathbf{9 \%}$ \\
\hline
\end{tabular}

\section{CONCLUSIONS}

In this paper, we have presented the highly efficient IPT system based on the co-design of the class-E power amplifier and a pair of Helical stacked coils for through-metal-wall power transfer. Measurement results show that the 200-Hz class-E PA achieves the peak power gain of $25.2 \mathrm{~dB}$ and the maximum collector efficiency of $57.3 \%$. The proposed IPT system has power delivery of $5 \mathrm{~W}$ and peak PTE of $9 \%$ for power transfer through the 3.1-mm thick aluminum barrier. 


\section{ACKNOWLEDGEMENT}

This work has been supported by Lawrence Livermore National Laboratory under the project number B620352.

\section{REFERENCES}

[1] D.-X. Yang, Z. Hu, H. Zhao, H.-F. Hu, Y.-Z. Sun and B.-J. Hou, ’Through-Metal-Wall Power Delivery and Data Transmission for Enclosed Sensors: A Review," MDPI Sensors Journal, vol. 15, pp. 31581-31605, December 2015.

[2] Y. Hu, X. Zhang, J. Yang, Q. Jiang, "Transmitting electric energy through a metal wall by acoustic waves using piezoelectric transducers," IEEE Transactions on Ultrasonics, Ferroelectrics, and Frequency Control, vol. 50, no. 7, pp. 773-781, July 2003.

[3] X. Bao, W. Biederman, S. Sherrit, M. Badescu, Y. Bar-Cohen, C. Jones, J. Aldrich, Z. Chang, ”Highpower piezoelectric acoustic-electric power feedthru for metal walls," The International Society for Optical Engineering (SPIE), vol. 6930, March 2008.

[4] D. J. Graham, J. A. Neasham and B. S. Sharif, "Investigation of Methods for Data Communication and Power Delivery Through Metals," IEEE Transactions on Industrial Electronics, vol. 58, no. 10, pp. 49724980, October 2011.

[5] H. Zangl, A. Fuchs, T. Bretterklieber, M. J. Moser and G. Hollerl, "Wireless Communication and Power Supply Strategy for Sensor Applications Within Closed Metal Walls," IEEE Transaction on Instrumentation and Measurement, vol. 59, no. 6, pp. 1686-1692, June 2010.

[6] L. Huang, A. P. Hu, A. Swain, S. Kim, Y. Ren, "An overview of capacitively coupled power transfer-A new contactless power transfer solution," 8th IEEE Conference on Industrial Electronics and Applications (ICIEA), pp. 461-465, June 2013.

[7] C. Liu, A. P. Hu, B. Wang, N. C. Nair, "A Capacitively Coupled Contactless Matrix Charging Platform with Soft Switched Transformer Control," IEEE Transaction on Industrial Electronics, vol. 60, no. 1, pp. 249-260, January 2013.

[8] M. Yamakawa, Y. Mizuno, J. Ishida, K. Komurasaki and H. Koizumi, ”Wireless Power Transmission into a Space Enclosed by Metal Walls Using Magnetic Resonance Coupling," Journal of Wireless Engineering and Technology, vol. 5, no. 1, pp. 19-24, January 2014.

[9] C. V. Pham, T. A. Vu, W. Tran, A.-V. Pham and C. S. Gardner, "Wireless Energy Harvesting System Through Metal for Aerospace Sensor,' 2018 IEEE Transportation Electrification Conference and Expo (ITEC), pp. 545-549, June 2018.

[10] C. V. Pham, A.-V. Pham and C. S. Gardner, "Development of Helical Circular Coils for Wireless Throughmetal Inductive Power Transfer," IEEE Wireless Power Transfer Conference (WPTC), pp. 1-3, May 2017.

[11] M. Kiani and M. Ghovanloo, "The circuit theory behind coupled-mode magnetic resonance-based wireless power transmission," IEEE Transaction on Circuits and Systems I: Regular papers, vol. 59, no. 9, pp. 2065-2074, September 2012.

[12] N. O. Sokal, "Class-E switching-mode high-efficiency tuned RF/microwave power amplifier: improved design equations," IEEE International Microwave Symposium (IMS), pp. 779-782, June 2000.

[13] F. H. Raab and N. O. Sokal, "Transistor Power Losses in the Class E Tuned Power Amplifier," IEEE Journal of Solid-State Circuits, vol. 13, no. 6, pp. 912-914, December 1978.

\section{BIOGRAPHIES OF AUTHORS}

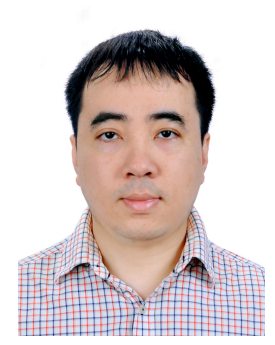

Tuan Anh Vu received the B.S degree and M.Sc degree in Electronics and Telecommunications Technology from University of Engineering and Technology, Vietnam National University in 2006 and 2009, respectively. In 2013, he received Ph.D degree in the field of analog/mixed-signal RF nanoelectronics from University of Oslo, Norway. Since 2014, he has been a lecturer at Faculty of Electronics and Telecommunications, VNU University of Engineering and Technology. Dr. Tuan Anh Vu was with Department of Semiconductor Electronics and Integration Science, Hiroshima University as a postdoctoral researcher for one year. He is now doing postdoc at Department of Electrical and Computer Engineering, University of California, Davis. His research interests are energy harvesting and analog RF integrated circuit designs including power amplifiers, low noise amplifiers, mixers, frequency multipliers, etc. 


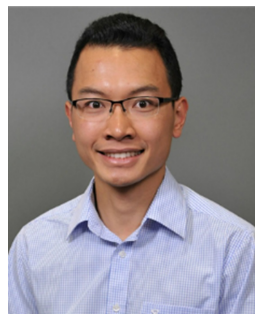

Chi Van Pham received the B.E. (with highest Hons.) degree in electronics and telecommunications engineering from Hanoi University of Science and Technology, Hanoi, Vietnam, in 2012, and the M.S. and Ph. D degrees in electrical and computer engineering from the University of California, Davis in 2015 and 2018, respectively. In 2018, he worked as RF/EM Design Engineer at Mobile Solutions (MSB) team at Skyworks Solutions, Inc. Then, he joined Apple Inc. as RF Design Engineer. His current research interest includes RF Front-end design, signal integrity, wide-band passive devices and antenna design, specifically the development of high power components and systems for radars and energy harvesting applications. Mr. Pham is a fellow of the Vietnam Education Foundation. He is the recipient of the prestigious IEEE MTT-S Fellowship Award in 2016.

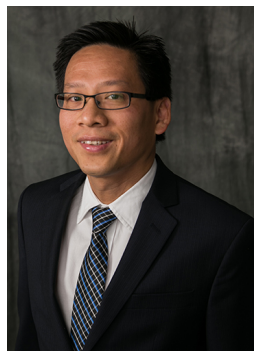

Anh-Vu Pham received the B.E.E. (with highest honors), M.S., and Ph.D. degrees in electrical engineering from the Georgia Institute of Technology, Atlanta, in 1995, 1997, and 1999, respectively. Anh- $\mathrm{Vu}$ joined the University of California at Davis in 2002 as an Assistant Professor and was promoted to full Professor in 2008. From 1999 to 2002, he was an Assistant Professor at Clemson University. Anh-Vu is conducting research in microwave and millimeter wave integrated circuit design, power amplifiers, electronic packaging, sensors, energy harvesting and phased array antennas. His research has been supported by DARPA, NSF, AFRL and numerous companies. He has published 140 peer-reviewed papers, several book chapters, and two books. Anh-Vu served as the Chair of IEEE Microwave Theory and Techniques (MTT) Technical Coordinate Committee on Microwave and Millimeter Packaging (2003-2006), and Chair of IEEE International Microwave Symposium Technical Committee on Power Amplifiers and Integrated Devices (2008-2010, 2012). He received the National Science Foundation CAREER Award in 2001 and the 2008 Outstanding Young Engineer Award from the IEEE Microwave Theory and Techniques Society. He was a Microwave Distinguished Lecturer of the IEEE MTT for the term 2010-2012. He is the Co-Chair of the Technical Program Committee for the IEEE International Microwave Symposium in San Francisco, 2016. Anh-Vu is currently a codirector of the Davis Millimeter Wave Research Center. Anh-Vu co-founded RF Solutions, a fabless semiconductor company providing power amplifiers and RFICs for WiFi applications. RF Solutions was acquired by Anadigics in 2003. In 2008, he co-founded and served as the Chief Technology Officer of Planarmag, Inc which was acquired by TE Connectivity in 2010. 